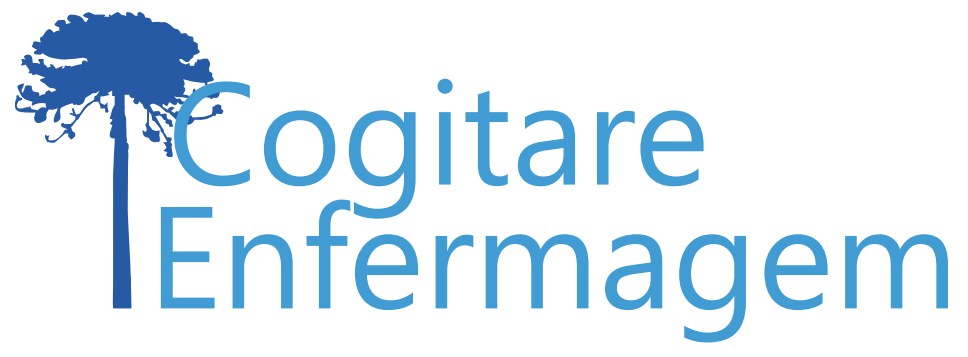

\title{
QUALIDADE DE VIDA DE PESSOAS AFETADAS PELA HANSENIIASE INSERIDAS EM GRUPOS DE APOIO AO AUTOCUIDADO*
}

Stephanie Steremberg Pires D'Azevedo', Danielle Christine Moura dos Santos², Maria Geórgia Torres Alves ${ }^{3}$, Niedja Madelon Nascimento Sousa4, Gustavo Aires de Arruda ${ }^{5}$, Marize Conceição Ventin Lima ${ }^{6}$

\section{RESUMO}

Objetivo: analisar a qualidade de vida de pessoas afetadas pela hanseníase inseridas em grupos de apoio ao autocuidado.

Método: estudo quantitativo, descritivo, com 43 participantes em unidades de referência que possuíam grupos de apoio ao autocuidado em hanseníase, em Recife e Região Metropolitana. A coleta de dados ocorreu entre janeiro e julho de $2018 \mathrm{com}$ a aplicação do instrumento de avaliação de qualidade de vida WHOQOL-bref. A distribuição das variáveis numéricas contínuas foi analisada pelo teste de Kolmogorov-Smirnov, as categóricas foram apresentadas por frequência absoluta e relativa (\%).

Resultados: o escore global de qualidade de vida foi 56,4 e os escores dos domínios revelaram-se medianos, com faixas de 50-60. Verificou-se que os domínios com menores pontuações foram os domínios físicos e relações ambientais.

Conclusão: pessoas afetadas pela hanseníase apresentam diminuição da qualidade de vida. Apesar de ter cura, é problema de saúde pública, necessitando de atençăo para controle e eliminação.

DESCRITORES: Qualidade de Vida; Hanseníase; Autocuidado; Educação; Mycobacterium leprae.

*Artigo extraído da dissertação de mestrado "Incapacidade funcional e qualidade de vida de pessoas atingidas pela hanseníase". Universidade de Pernambuco, 2019.

COMO REFERENCIAR ESTE ARTIGO:

D'Azevedo SSP, Santos DCM dos, Alves MGT, Sousa NMN, Arruda GA de, Lima MCV. Qualidade de vida de pessoas afetadas pela hanseníase inseridas em grupos de apoio ao autocuidado. Cogitare enferm. [Internet]. 2019 [acesso em "colocar data de acesso, dia, mês abreviado e ano"]; 24. Disponível em: http://dx.doi.org/10.5380/ ce.v24i0.64266.

Este obra está licenciado com uma Licença Creative Commons Atribuição 4.0 Internacional.

${ }^{1}$ Enfermeira. Mestranda em Enfermagem. Universidade de Pernambuco e Universidade Estadual da Paraíba. Recife, PE, Brasil. 9

${ }^{2}$ Enfermeira. Doutora em Saúde Coletiva. Docente de Enfermagem da Universidade de Pernambuco. Recife, PE, Brasil. [ ${ }^{3}$ Discente de Enfermagem. Universidade de Pernambuco. Recife, PE, Brasil. 9

${ }^{4}$ Discente de Enfermagem. Universidade de Pernambuco. Recife, PE, Brasil. $\bigcirc$

${ }^{5}$ Profissional de Educação Física. Doutor em Educação Física. Universidade de Pernambuco e Universidade Estadual da Paraíba. Recife, PE, Brasil. $($ )

${ }^{6}$ Enfermeira. Mestranda em Enfermagem. Universidade de Pernambuco e Universidade Estadual da Paraíba. Recife, PE, Brasil. 


\title{
QUALITY OF LIFE OF PEOPLE AFFECTED BY LEPROSY IN SELF- CARE SUPPORT GROUPS
}

\author{
ABSTRACT \\ Objective: To analyze the quality of life of people affected by leprosy in self-care support \\ groups. \\ Method: A quantitative and descriptive study conducted with 43 participants in reference units \\ with self-care support groups for people affected by leprosy in Recife and its Metropolitan \\ Area. Data collection took place between January and July 2018 with the application of \\ the WHOQOL-BREF instrument to assess quality of life. The distribution of the continuous \\ numerical variables was analyzed by the Kolmogorov-Smirnov test; the categorical variables \\ were presented by absolute and relative (\%) frequency. \\ Results: The overall quality of life score was 56.4 and the domain scores were average, ranging \\ between 50 and 60 . The domains with the lowest scores were found to be the physical and \\ environmental relationship domains \\ Conclusion: People affected by leprosy have a decreased quality of life. Although curable, it \\ is a public health problem and requires attention for control and elimination.
}

DESCRIPTORS: Quality of Life; Leprosy; Self-care; Education; Mycobacterium leprae.

\section{CALIDAD DE VIDA DE PERSONAS CON LEPRA QUE ACUDEN A GRUPOS DE APOYO PARA EL AUTOCUIDADO}

\begin{abstract}
RESUMEN:
Objetivo: analizar la calidad de vida de personas con lepra que acuden a grupos de apoyo para el autocuidado.

Método: estudio cuantitativo y descriptivo realizado con 43 participantes en unidades de referencia que poseían grupos de apoyo para el autocuidado de la lepra, en Recife y en su Región Metropolitana. Los datos se recolectaron entre enero y julio de 2018 con la aplicación del instrumento de evaluación de la calidad de vida llamado WHOQOL-bref. La distribución de las variables numéricas continuas se analizó con la prueba de Kolmogorov-Smirnov; las categóricas se presentaron por medio de frecuencias absolutas y relativas (\%).

Resultados: el puntaje global de la calidad de vida fue 56,4 y los de los dominios resultaron medianos, con un margen de 50 a 60 . Se verificó que los dominios con los puntajes más bajos fueron los dominios físico y de relaciones ambientales.

Conclusión: las personas con lepra presentan una reducción en su calidad de vida. Pese a tener cura, es un problema de salud pública, que requiere atención para su control y eliminación.
\end{abstract}

DESCRIPTORES: Calidad de vida; Lepra; Autocuidado; Educación; Mycobacterium leprae. 
A hanseníase constitui-se uma doença infectocontagiosa, de evolução lenta, progressiva, curável, de caráter crônico, cujo agente etiológico é o Mycobacterium leprae, bacilo de alta infectividade e baixa patogenicidade(1). A transmissão da doença se dá por meio das vias respiratórias, por contato prolongado com pacientes infectados e sem tratamento adequado(1).

Considerada uma doença negligenciada e milenar, a hanseníase possui uma relação estreita entre as condições de vida dos indivíduos afetados por ela e o processo de saúde e doença $a^{(2)}$. Segundo a Organização Mundial de Saúde (OMS) $)^{(3)}$, ainda é problema de saúde públiça. O diagnóstico apresenta algumas dificuldades, pois, muitas vezes o quadro clínico é confundido com outras doenças de pele, situação que favorece o diagnóstico tardio dos casos e, consequentemente, a ocorrência de incapacidades físicas ${ }^{(3)}$.

As incapacidades e deformidades causadas pela hanseníase excedem o domínio físico e afetam a qualidade de vida dos doentes, com evidentes obstáculos na realização de atividades de vida diária, falta de interação do indivíduo com a comunidade e impactos psicológicos ${ }^{(4-5)}$.

Nos últimos anos, estudos internacionais destacam a qualidade de vida de pessoas afetadas pela hanseníase, com ênfase no empoderamento do paciente e foco na redução de estigma, programas de reabilitação, integração social e diagnóstico precoce para minimizar a limitação de atividade, restrição de participação social e melhoria na qualidade de vida ${ }^{(6-9)}$.

A qualidade de vida das pessoas afetadas pela hanseníase pode ser prejudicada devido às alterações físicas e deformidades ocasionadas pela doença, acarretando mudanças na percepção do indivíduo de ver o seu próprio corpo, e como a comunidade o percebe, gerando grande impacto psicossocial ${ }^{(10-14)}$.

Diante disso, todas as estratégias que visem a prevenção e o tratamento das incapacidades e das deformidades são essenciais para que a pessoa afetada pela doença consiga melhorar sua qualidade de vida(15). Uma das estratégias é a promoção do autocuidado: ações e atividades que o próprio paciente realiza, entendendo os riscos e promovendo por meio de medidas a promoção, a prevenção e a recuperação da sua saúde. Dentre as abordagens ao autocuidado, pode-se citar os grupos de apoio ao autocuidado ${ }^{(16-17)}$.

Diante da importância da compreensão dos fatores determinantes da doença e da existência de instrumentos validados que auxiliam a mensuração de indicadores da qualidade de vida, este estudo objetivou analisar a qualidade de vida de pessoas afetadas pela hanseníase inseridas em grupos de apoio ao autocuidado.

\section{MÉTODO}

Tratou-se de um estudo quantitativo, descritivo. A pesquisa foi realizada no período de janeiro a julho de 2018, com pessoas afetadas pela hanseníase que estão inseridas em Grupos de Apoio ao Autocuidado em Hanseníase (GAC) das unidades de referência municipais ou estaduais localizadas na cidade de Recife-PE e na Região Metropolitana.

Foram incluídos todos os seis GAC que realizavam reuniões periódicas. A população do estudo envolvia os membros dos GAC, que não possuía número fixo de participantes, pois os grupos têm acesso aberto, permitindo assim a entrada ou saída dos seus membros a qualquer momento. 
O processo de amostragem foi realizado de forma não probabilística (conveniência / acessibilidade). No momento do início da pesquisa havia um total de 70 participantes cadastrados nos grupos de apoio. Durante a coleta de foi obtido um total de 43 participantes, o que corresponde a $61,4 \%$ do total. Para este estudo, os pesquisadores estiveram presentes num total de 30 reuniões. Foram coletados em média os dados de sete participantes por reunião.

A coleta de todos os participantes não foi possível, uma vez que durante o período de coleta ocorreram ausências.

O cálculo do tamanho amostral foi realizado com o programa G*Power versão 3.1.9.2. Ao considerar os resultados obtidos ${ }^{(14)}$ para a questão "Como você avaliaria sua qualidade de vida?", sendo as frequências: "muito ruim" $=0,0 \%$, "ruim" $=0,0 \%$, "nem ruim nem boa" $=38,8 \%$, "boa" $=52,7 \%$ e "muito boa" $=8,3 \%$, considerando um alfa de $5 \%$ e, poder de $80 \%$, o tamanho amostral mínimo necessário para este estudo seria de 11 participantes.

Os critérios de inclusão foram: ser pessoa afetada pela hanseníase e participar do GAC. Foram excluídos do estudo os participantes com deficiência mental descrita em prontuário que impossibilitasse a compreensão do questionário, instrumentos aplicados, do tema e/ou objetivo da pesquisa, participantes que estavam em tratamento da doença em um período menor que três meses e pessoas menores de 18 anos.

A coleta de dados foi realizada por meio da aplicação de dois instrumentos, com a presença dos pesquisadores em ambiente reservado e tranquilo, sendo apenas um encontro suficiente para o preenchimento das informações (para cada participante, individualmente). O primeiro instrumento foi composto por questões sociodemográficas e econômicas e dados clínicos, estes, coletados por meio de prontuários dos participantes.

Em relação à percepção da qualidade de vida, foi utilizado o instrumento de avaliação de qualidade de vida da Organização Mundial de Saúde - World Health Organization Quality of Life (WHOQOL-bref(18) versão abreviada, validada, no idioma português. É composta por 26 perguntas, as duas primeiras correspondem aos aspectos gerais de qualidade de vida e as demais 24 são divididas em quatro domínios (físico, psicológico, relações sociais e meio ambiente), e as respostas seguem uma escala do tipo Likert(18).

A análise dos domínios foi realizada individualmente e em escore global de qualidade de vida. Cada domínio possui questões que variam de um a cinco pontos. Quanto maior a pontuação, maior a qualidade de vida, com exceção das questões três, quatro e 26 que possuem escore inverso. Os escores foram transformados para escala de 0-100 em pontuação(18).

A distribuição das variáveis numéricas contínuas foi analisada pelo teste de Kolmogorov-Smirnov. A apresentação dos resultados foi realizada mediante estatística descritiva. Os resultados das variáveis numéricas contínuas foram descritos por meio de medidas de tendência central (média e mediana) e dispersão (desvio-padrão e intervalo interquartil).

O teste binomial foi utilizado para a comparação de proporções em relação a uma distribuição aleatória para as variáveis sexo, classificação operacional e reação hansênica. As variáveis categóricas foram apresentadas por meio de frequência absoluta e relativa (\%). O teste de Qui-quadrado de aderência foi utilizado para a comparação das frequências observadas em relação às esperadas. A análise dos dados foi realizada com o programa SPSS Statistics for Windows, v 20.

A pesquisa foi aprovada pelo Comitê de Ética e Pesquisa com Seres Humanos do Complexo Hospitalar da Universidade de Pernambuco, sob parecer $\mathrm{n}^{\circ}$ 2.309.191. 
A amostra do estudo foi constituída por 43 pessoas. Os resultados sobre a caracterização sociodemográfica e econômica estão dispostos na Tabela 1.

Tabela 1 - Caracterização sociodemográfica e econômica de pessoas afetadas pela hanseníase $(n=43)$ inseridas em grupos de apoio ao autocuidado em Recife (PE) e na Região Metropolitana. Recife, PE, Brasil, 2018 (continua)

\begin{tabular}{|c|c|c|c|}
\hline Variável & $\mathbf{n}$ & $\%$ & $\mathbf{p}$ \\
\hline \multicolumn{4}{|l|}{ Sexo } \\
\hline Masculino & 23 & 53,5 & \\
\hline Feminino & 20 & 46,5 & 0,761 \\
\hline \multicolumn{4}{|l|}{ Idade } \\
\hline 20-39 anos & 6 & 14 & \\
\hline $40-59$ anos & 20 & 46,5 & 0,023 \\
\hline 60 anos ou mais & 17 & 39,5 & \\
\hline \multicolumn{4}{|l|}{ Etnia } \\
\hline Parda & 23 & 53,5 & \\
\hline Preta & 12 & 28 & $<0,001$ \\
\hline Branca & 7 & 16,3 & \\
\hline Outros & 1 & 2,2 & \\
\hline \multicolumn{4}{|l|}{ Procedência } \\
\hline Recife & 15 & 35 & \\
\hline Região Metropolitana & 5 & 11,5 & 0,003 \\
\hline Outros & 23 & 53,5 & \\
\hline \multicolumn{4}{|l|}{ Estado civil } \\
\hline Solteiro & 15 & 35 & \\
\hline Casado & 14 & 32,6 & 0,141 \\
\hline União estável & 6 & 14 & \\
\hline Outros & 8 & 18,4 & \\
\hline \multicolumn{4}{|l|}{ Status de trabalho } \\
\hline Aposentado & 15 & 35 & \\
\hline Autônomo & 4 & 9,3 & \\
\hline Do lar & 10 & 23,3 & 0,062 \\
\hline Desempregado & 5 & 11,6 & \\
\hline Outros & 9 & 20,8 & \\
\hline \multicolumn{4}{|l|}{ Renda mensal } \\
\hline Sem renda & 4 & 9,2 & \\
\hline 1 salário mínimo & 6 & 14 & $<0,001$ \\
\hline 2-3 salários mínimos & 27 & 62,8 & \\
\hline Mais de 3 salários mínimos & 6 & 14 & \\
\hline
\end{tabular}




\begin{tabular}{lllll}
\hline Escolaridade & & & \multirow{2}{*}{0,039} \\
\cline { 1 - 3 } 1-3 anos de estudo & 9 & 21 & \\
\cline { 1 - 3 } 4-7 anos de estudo & 22 & 51 & \\
\cline { 1 - 3 } 8 anos ou mais de estudo & 12 & 28 & \\
\cline { 1 - 3 } Moradia & & & \\
\cline { 1 - 4 } Própria & 37 & 86 & $<0,001$ \\
\hline Alugada & 4 & 9,3 & \\
\cline { 1 - 3 } Outros & 2 & 4,7 &
\end{tabular}

Em relação à classificação operacional, a predominância foi a multibacilar (MB) que correspondeu a $37(90,2 \%)$ participantes seguida pela paucibacilar (PB) com quatro $(9,8 \%)$ participantes. Em relação às formas clínicas, observou-se: 19 (50\%) pessoas com a forma virchowiana, $18(47,4 \%)$ participantes com a forma clínica dimorfa e uma $(2,6 \%)$ pessoa com a forma clínica tuberculoide.

Em relação aos graus de incapacidade, $18(47,4 \%)$ pessoas foram diagnosticadas com grau II, $14(36,8 \%)$ participantes com presença de grau l e seis $(15,8 \%)$ pessoas não tinham incapacidades (grau zero) no momento do diagnóstico. Apesar de $32(84,2 \%)$ participantes já possuírem algum grau de incapacidade no momento da avaliação inicial, a frequência de resposta não foi significativamente diferente entre as classificações $(p=0,052)$, isso também ocorreu para a reação hansênica, sendo identificada em $22(57,2 \%)$ participantes $(p=0,418)$. Já o uso de órtese/prótese ocorreu em menor proporção, sendo relatado por apenas cinco $(17,2 \%)$ pessoas $(p=0,001)$.

Ao serem abordados diretamente, com questões do instrumento relacionadas aos aspectos gerais de qualidade de vida, têm-se os seguintes enfoques, "Como você avaliaria sua qualidade de vida? e "Quão satisfeito(a) você está com sua saúde?", em relação às duas semanas anteriores, apenas $14(32,6 \%)$ pessoas referiram perceber sua qualidade de vida como "boa" e duas pessoas (4,7\%) como "muito boa". Mais da metade da amostra, $22(51 \%)$ pessoas referiram como "nem ruim nem boa" e cinco $(11,7 \%)$ participantes responderam como "ruim" ( $p<0,001)$.

Ao analisar a satisfação em relação à sua saúde, apenas um $(2,3 \%)$ participante disse estar "muito satisfeito", 11 (25,6\%) pessoas disseram que estavam "satisfeitas", 19 (44,2\%) pessoas "nem satisfeitos nem insatisfeitos", sete $(16,3 \%)$ pessoas "insatisfeitas" e cinco $(11,6 \%)$ participantes estavam "muito insatisfeitos". Em ambas as questões, a distribuição foi diferente da esperada aleatoriamente ( $20 \%$ por alternativa), porém $11,6 \%$ e $27,9 \%$ indicaram condições que sugerem a necessidade de melhoria para a saúde e qualidade de vida, respectivamente. O instrumento WHOQOL-bref possui quatro domínios: físico, psicológico, relações sociais e meio ambiente. Os escores dos domínios estão apresentados na Tabela 2.

Tabela 2 - Escore dos domínios de qualidade de vida de pessoas afetadas pela hanseníase $(n=43)$ inseridas em grupos de apoio ao autocuidado em Recife (PE) e Região Metropolitana. Recife, PE, Brasil, 2018 (continua)

\begin{tabular}{lccccc} 
Domínios WHOQOL-bref & Média & DP & Mediana & $\mathbf{1}^{\circ}$ quartil & $\mathbf{3}^{\circ}$ quartil \\
\hline Físico & 50,5 & 21,8 & 57,1 & 32,1 & 64,3 \\
\hline Psicológico & 62,1 & 16,1 & 66,6 & 54,2 & 70,8 \\
\hline
\end{tabular}




\begin{tabular}{lccccc}
\hline Relações sociais & 62,5 & 22,4 & 66,6 & 50 & 75 \\
\hline Meio ambiente & 50,5 & 13,8 & 50 & 43,8 & 59,4
\end{tabular}

O escore global de qualidade de vida para os participantes foi 56,4 (desvio padrão $=15$; Mediana $=58,1$; Intervalo interquartil $=46,7-65,7$ ) e os escores dos domínios analisados revelaram-se medianos com faixas de 50-60, sugerindo que nenhum domínio específico foi mais prejudicado pela doença (Tabela 2). Os resultados do domínio físico estão apresentados na Tabela 3.

Tabela 3 - Domínio físico de qualidade de vida de pessoas afetadas pela hanseníase $(n=43)$ inseridas em grupos de apoio ao autocuidado em Recife (PE) e Região Metropolitana. Recife, PE, Brasil, 2018

WHOQOL-bref - Domínio Físico

\begin{tabular}{lcccccc}
\hline Facetas (\%) & Nada & $\begin{array}{c}\text { Muito } \\
\text { pouco }\end{array}$ & $\begin{array}{c}\text { Mais ou } \\
\text { Menos }\end{array}$ & Bastante & $\begin{array}{c}\text { Extrema- } \\
\text { mente }\end{array}$ & p \\
\hline Dor e desconforto & 14 & 32,5 & 20,9 & 18,6 & 14 & 0,285 \\
\hline $\begin{array}{l}\text { Dependência de } \\
\text { medicamento ou tratamento }\end{array}$ & 16,3 & 46,5 & 25,6 & 9,3 & 2,3 & $<0,001$ \\
\hline Facetas (\%) & Nada & $\begin{array}{c}\text { Muito } \\
\text { pouco }\end{array}$ & Médio & Muito & $\begin{array}{c}\text { Completa- } \\
\text { mente }\end{array}$ & p \\
\hline Energia e fadiga & 7 & 16,3 & 20,9 & 44,2 & 11,6 & 0,001 \\
\hline Facetas (\%) & Muito & Ruim & Nem ruim & Bom & $\begin{array}{c}\text { Muito } \\
\text { bom }\end{array}$ & p \\
\hline Mobilidade & 4,7 & 16,3 & 23,3 & 39,5 & 16,2 & 0,007 \\
\hline Sono e repouso & 14 & 18,5 & 14 & 39,5 & 14 & 0,031 \\
\hline Atividades de vida cotidiana & 14 & 11,6 & 27,9 & 37,2 & 9,3 & 0,014 \\
\hline Capacidade de trabalho & 20,9 & 25,6 & 18,6 & 27,9 & 7 & 0,221
\end{tabular}

O domínio psicológico apresentou escore com maior pontuação $(62,1)$ em relação ao domínio físico $(50,5)$. Um dado relevante no domínio psicológico envolve a questão que abordava sobre o quanto aproveitam a vida, mais de $60,5 \%$ dos participantes relataram que aproveitam "nada" e "muito pouco". A maior parte das pessoas $90,6 \%$ percebeu que tiveram sentimentos negativos, tais como mau humor, desespero, ansiedade e depressão, "frequentemente", "muito frequentemente" ou "sempre" durante as duas últimas semanas.

Observou-se que os participantes não perceberam como prejudicados em relação ao relacionamento com amigos, parentes, conhecidos, atividade sexual e suporte (apoio social). A seguir, a Tabela 4 traz os resultados do domínio das relações sociais. 
Tabela 4 - Domínio das relações sociais de qualidade de vida de pessoas afetadas pela hanseníase $(n=43)$ inseridas em grupos de apoio ao autocuidado em Recife (PE) e Região Metropolitana. Recife, PE, Brasil, 2018

WHOQOL-bref - Domínio Relações sociais

\begin{tabular}{lcccccc}
\hline Facetas (\%) & $\begin{array}{c}\text { Muito } \\
\text { insatisfeito }\end{array}$ & Insatisfeito & $\begin{array}{c}\text { Nem } \\
\text { satisfeito } \\
\text { nem } \\
\text { insatisfeito }\end{array}$ & Satisfeito & $\begin{array}{c}\text { Muito } \\
\text { satisfeito }\end{array}$ & P \\
\hline Relações pessoais & 2,3 & 16,3 & 20,9 & 39,5 & 21 & 0,004 \\
\hline Atividade sexual & 9,3 & 11,6 & 16,3 & 48,8 & 14 & $<0,001$ \\
\hline Suporte (apoio) social & 11,6 & 4,7 & 25,6 & 44,1 & 14 & $<0,001$
\end{tabular}

Foi observado que, ao relatarem sobre a sua segurança física e proteção, $25,6 \%$ perceberam "nada" e "muito pouco", respectivamente, a distribuição não sendo diferente da esperada aleatoriamente $(p=0,099)$. Os participantes foram questionados sobre a satisfação com o seu meio de transporte e $37 \%$ afirmaram estarem "insatisfeitos" ou "muito insatisfeitos" ( $p=0,008)$. Ao serem questionados sobre as oportunidades de atividades de lazer, 55,8\% dos participantes relataram "nada" ou "muito pouco", a frequência ocorrendo de forma significativamente $(p<0,001)$ diferente da esperada aleatoriamente (Tabela 5).

Tabela 5 - Domínio das relações ambientais de qualidade de vida de pessoas afetadas pela hanseníase $(n=43)$ inseridas em grupos de apoio ao autocuidado em Recife (PE) e Região Metropolitana. Recife, PE, Brasil, 2018

WHOQOL-bref - Domínio meio ambiente

\begin{tabular}{|c|c|c|c|c|c|c|}
\hline Facetas (\%) & Nada & $\begin{array}{l}\text { Muito } \\
\text { pouco }\end{array}$ & $\begin{array}{c}\text { Mais ou } \\
\text { menos }\end{array}$ & Bastante & $\begin{array}{l}\text { Extrema- } \\
\text { mente }\end{array}$ & $\mathbf{p}$ \\
\hline Segurança física e proteção & 11,6 & 14 & 32,5 & 27,9 & 14 & 0,099 \\
\hline Ambiente no lar & 0 & 14 & 32,6 & 39,4 & 14 & 0,032 \\
\hline $\begin{array}{l}\text { Ambiente físico: poluição/ruído/ } \\
\text { trânsito }\end{array}$ & 7 & 7 & 23,3 & 53,5 & 9,2 & $<0,001$ \\
\hline Facetas (\%) & Nada & $\begin{array}{l}\text { Muito } \\
\text { pouco }\end{array}$ & Médio & Muito & $\begin{array}{l}\text { Completa- } \\
\text { mente }\end{array}$ & $\mathbf{p}$ \\
\hline Recursos financeiros & 23,3 & 41,8 & 27,9 & 4,7 & 2,3 & $<0,001$ \\
\hline $\begin{array}{l}\text { Oportunidades de adquirir novas } \\
\text { informações e habilidades }\end{array}$ & 9,3 & 27,9 & 32,6 & 25,5 & 4,7 & 0,012 \\
\hline $\begin{array}{l}\text { Participação em, e oportunidades } \\
\text { de recreação e lazer }\end{array}$ & 9,3 & 46,5 & 23,3 & 16,3 & 4,6 & $<0,001$ \\
\hline $\begin{array}{l}\text { Cuidados de saúde e sociais: } \\
\text { disponibilidade e qualidade }\end{array}$ & 7 & 25,6 & 14 & 46,4 & 7 & \\
\hline Transporte & 9 & 28 & 18,6 & 37,2 & 7,2 & 0,008 \\
\hline
\end{tabular}


Sobre a situação financeira, $65,1 \%$ dos participantes referiram ter "nada" ou "muito pouco", dinheiro para satisfazer suas necessidades, o que corrobora com a caracterização da renda destes participantes, que estavam na faixa de dois a três salários mínimos $(62,8 \%)$, em sua predominância. Quanto à disponibilidade de informações para o dia a dia, 37,2\% referem algum grau de restrição, "nada" ou "muito pouco" para acessar as informações com distribuição sendo diferente da esperada aleatoriamente $(p=0,012)$, o que pode estar relacionado à falta de informação e/ou ao nível de escolaridade da população estudada que em sua maior parte, $51 \%$ têm entre quatro a sete anos de estudo (Tabela 5 ).

\section{DISCUSSÃO}

Neste estudo, foi possível verificar que as pessoas afetadas pela hanseníase apresentam diminuição na sua qualidade de vida. Destaca-se que os participantes apresentaram o escore global de qualidade de vida de 56,4 , e os escores dos domínios analisados revelaram-se medianos com faixas de 50-60. Identificou-se que a presença da doença pode estar relacionada ao prejuízo na qualidade de vida geral. Ao analisar cada domínio, verificou-se que os domínios com menores pontuações em relação aos demais foram os domínios físico, 50,5 e meio ambiente, 50,5.

Em relação à caracterização da população, a sua maior parte foi do sexo masculino $53,5 \%$, o que corrobora em relação ao gênero em outros estudos ${ }^{(10-11)}$, onde $54,3 \%$ e $62,1 \%$, respectivamente. A maior exposição ao bacilo e o menor cuidado de indivíduos do sexo masculino com a saúde também foram citados, o que leva ao retardo do diagnóstico e aumenta o risco para o desenvolvimento de incapacidades físicas ${ }^{(19)}$.

Quanto ao grau de escolaridade e renda, os dados deste estudo sugerem maior fragilidade da população afetada pela hanseníase e convergem com achados em alguns estudos $^{(10,12)}$, sobre a caracterização de pessoas atingidas pela doença no Brasil. Essas informações podem dificultar e contribuir de maneira negativa na compreensão sobre a doença, o diagnóstico, o tratamento e o autocuidado por parte dos participantes, e ressaltam os prejuízos econômicos para os mesmos, suas famílias e a sociedade ${ }^{(10,12)}$.

Neste estudo predominou a faixa etária entre $40-59$ anos (46,5\%), e participantes aposentados (35\%). Estudos de dois estados do Brasil, na Paraíba $(100,0 \%)^{(12)}$ e em Minas Gerais $(68 \%)^{(20)}$ corroboraram com esta caracterização socioeconômica(12,21). Devido ao diagnóstico tardio e a presença de incapacidades, as pessoas necessitam de afastamento de suas atividades laborais devido às complicações ocasionadas pela doença.

Quanto à classificação operacional, forma clínica e grau de incapacidade, a presença da forma multibacilar é a mais predominante nas populações estudadas, assim como neste estudo $90,2 \%$, do tipo virchowiana $47,4 \%$. Para fins operacionais de tratamento, os doentes são classificados em paucibacilares (PB - até cinco lesões de pele com baciloscopia de raspado intradérmico negativo) ou multibacilares (MB - seis ou mais lesões de pele ou baciloscopia de raspado intradérmico positiva) $)^{(1)}$.

Estudos sobre o perfil das pessoas afetadas pela hanseníase discutiram sobre a relação entre o alto diagnóstico de casos multibacilares e diagnóstico tardio, visto que se configura como doença sistêmica, que atinge diversos órgãos, e é a forma clínica mais grave e potencialmente infectante $e^{(1,19,22)}$.

Avaliar o grau de incapacidade é de extrema importância na elaboração de estratégias para a prevenção de incapacidades e da construção do autocuidado(1). Mais de $84,2 \%$ dos participantes deste estudo foram avaliados com graus de incapacidade I e II na avaliação diagnóstica. Diferentemente de outro estudo ${ }^{(14)}$, que apontou a presença de incapacidades físicas instaladas com predomínio de $62 \%$ da população. Diante disso, destaca-se que a amostra era composta por participantes de grupos de autocuidado, e que isso pode ter influenciado o perfil dos participantes do estudo, já que em algumas unidades os grupos 
são voltados para os indivíduos que já possuem alguma incapacidade.

O domínio físico de qualidade de vida apresentou escore baixo $(50,1)$, e pode ser consequência dos efeitos da doença, evidenciados nas incapacidades físicas. Estudos brasileiros também apresentam escores menores neste domínio ${ }^{(11-13)}$.

A percepção dos participantes no domínio das relações sociais de qualidade de vida não demostrou relação com diminuição de qualidade de vida percebidas por eles, com média de escore de 62,5. O fato de os participantes estarem inseridos dentro de um grupo de apoio ao autocuidado, onde compartilhavam experiências e vivenciavam dificuldades juntos, pode colaborar positivamente para percepção deles em relação às amizades e ao relacionamento com os pares.

No domínio psicológico de qualidade de vida, mesmo a despeito de a média do escore ter sido mais alta do que nos demais domínios prejudicados $(62,1)$, mais de $90 \%$ dos participantes relataram possuírem sentimentos negativos. Em um estudo ${ }^{(12)}$ o domínio psicológico contribuiu com diminuição da qualidade de vida e as pessoas afetadas pela doença relataram que as modificações físicas ocasionadas pela doença diminuíam sua autoestima, autopercepção e qualidade de vida, contribuindo negativamente para este domínio.

O domínio das relações ambientais pode contribuir para o prejuízo da qualidade de vida, pois envolve questões referentes à proteção física e social, ambiente do lar, recursos financeiros e transporte e, nessas questões, a maior parte percebeu como prejudicados, o que pode estar relacionado ao perfil socioeconômico dos participantes deste estudo, como foi encontrado em outros estudos ${ }^{(23-24)}$. Este domínio foi encontrado com a média de escore menor, 50,5, em relações aos demais domínios, achados também em estudos de Minas Gerais e do Paraná(13-14). Os participantes perceberam dificuldades (62,8\%) em relação à sua participação (atividades comunitárias).

Os resultados encontrados neste estudo podem proporcionar um direcionamento das ações dos profissionais de saúde e na reorganização do processo de trabalho dentro do atendimento individualizado e/ou em grupos, com o intuito de traçar metas e estratégias voltadas aos domínios prejudicados e nos determinantes que levam à diminuição da qualidade de vida destes indivíduos.

As limitações deste estudo estão relacionadas ao método de amostragem das pessoas afetadas pela hanseníase inseridas nos grupos de autocuidado e o próprio grupo, pois estes se comportam de maneira dinâmica e as pessoas participam mediante disponibilidade e interesse, o que dificultou o processo de coleta de dados. O delineamento transversal impossibilita possíveis visualizações sobre a causa e o efeito dos problemas identificados nos escores dos domínios de qualidade de vida, além do impacto da presença de grupos de apoio ao autocuidado na qualidade de vida e na prevenção de incapacidades nestas pessoas.

\section{CONCLUSÃO}

Os resultados encontrados evidenciam que a percepção de qualidade de vida dos participantes teve baixa pontuação. Vários problemas foram observados e relacionadoș à insatisfação da qualidade de vida percebidas pelas pessoas afetadas pela doença. É necessária uma visão ampliada, com perspectiva abrangente no cuidado e na compreensão dos determinantes que levam ao prejuízo da qualidade de vida, sempre os associando à clínica e aos aspectos psicossociais.

Destaca-se a necessidade da realização de mais estudos sobre esta temática, considerando a percepção destas pessoas em relação ao adoecimento, ao conceito de saúde-doença, e as repercussões dos grupos de autocuidado na vida dos seus membros. 
Agradecemos o apoio financeiro da organização não-governamental NHR Brasil (Netherlands Hanseniasis Relief - Brasil) para a realização deste estudo. Programa Nacional de Pós-Doutorado da CAPES (PNPD/CAPES).

\section{REFERÊNCIAS}

1. Ministério da Saúde (BR). Guia prático sobre a hanseníase. [Internet] Brasília: Ministério da Saúde; 2017 [acesso em 01 out 2018]. Disponível em: https://portalarquivos2.saude.gov.br/images/pdf/2017/ novembro/22/Guia-Pratico-de-Hanseniase-WEB.pdf.

2. Gonçalves M, Prado MAR do, Silva SS da, Santos K da S, Araújo PN de, Fortuna CM. Work and Leprosy: women in their pains, struggles and toils. Rev. bras. enferm. [Internet]. 2018 [acesso em 12 nov 2018]; 71(Suppl 1). Disponível em: http://dx.doi.org/10.1590/0034-7167-2017-0598.

3. World Health Organization (WHO). Estratégia Global para Hanseníase 20162020 [Internet]. Genebra: WHO; 2016 [acesso em 01 jun 2017]. Disponível em: https://apps.who.int/iris/bitstream/handle/10665/208824/9789290225201-pt. pdf;jsessionid=FF749A934CEA6E399A1F23674FAA2DE2? sequence=17.

4. Aishia F, Wiarsih W. Disability Grades and Quality of Social Relationships among People with Leprosy in Donorojo Leprosy Rehabilitation Village. Ul. Proc. Health. Med. [Internet]. 2017 [acesso em 02 jun 2018]; 3(1). Disponível em: http://proceedings.ui.ac.id/index.php/uiphm/article/view/32.

5. Toh HS, Maharjan J, Thapa R, Neupane KD, Shah M, Baral S, et al. Diagnosis and impact of neuropathic pain in leprosy patients in Nepal after completion of multidrug therapy. PLoS Negl Trop Dis [Internet]. 2018 [acesso em 15 fev 2019]; 12(7). Disponível em: https://doi.org/10.1371/journal.pntd.0006610.

6. Eyanoer PC. Social acceptance and quality of life of leprosy patient. IOP Conf. Series: Earth and Environmental Science [Internet]. 2018 [acesso em 25 fev 2019]; 125(2018). Disponível em: https://doi. org/10.1088/1755-1315/125/1/012100.

7. Govindharaj P, Srinivasan S, Darlong J. Quality of life of people affected with leprosy disability living in Purulia, West Bengal. Int. J. Health. Sci. Res [Internet]. 2018 [acesso em 22 fev 2019]; 8(2). Disponível em: http://www.ijhsr.org/IJHSR Vol.8 Issue.2 Feb2018/28.pdf.

8. Xiong M, Wang X, Su T, Yang B, Li M, Zheng D. Relationship between psychological health and quality of life of people affected by leprosy in the community in Guangdong province, China: a cross-sectional study. BMC Public Health [Internet]. 2019 [acesso em 26 jun 2019]; 19(1). Disponível em: https://doi. org/10.1186/s12889-019-6672-x.

9. Pereira EF, Teixeira CS, Santos A dos. Qualidade de vida: conceitos, abordagens e avaliação. Rev. bras. educ. fís. esport. [Internet]. 2012 [acesso em 18 nov 2018]; 26(2). Disponível em: http://dx.doi. org/10.1590/S1807-55092012000200007.

10. Simões S, Castro SS, Scatena LM, Castro RO, Lau FA. Qualidade de vida dos portadores de hanseníase. Medicina (Ribeirão Preto) [Internet]. 2016 [acesso em 15 nov 2018]; 49(1). Disponível em: https://doi.org/10.11606/issn.2176-7262.v49i1p60-67.

11. Araújo DAL, Brito KKG, Santana EMF, Soares VL, Soares MJGO. Caracterização da qualidade de vida de pessoas com hanseníase em tratamento ambulatorial. Rev. pesqui. cuid. fundam. (Online). [Internet]. 2016 [acesso em 18 mar 2018]; 8(4). Disponível em: http://dx.doi.org/10.9789/2175-5361.2016.v8i4.50105016.

12. Leite IF, Arruda AJCG de, Vasconcelos DIB de, Santana SC de, Chianca KSV. A qualidade de vida em pacientes com hanseníase crônica. Rev. enferm. UFPE on line [Internet]. 2015 [acesso em 29 out 2018]; 
9(6). Disponível em: https://periodicos.ufpe.br/revistas/revistaenfermagem/article/view/10574.

13. Dolenz MFA, Guerra-Silva N, Melo SCCS de, Tashima CM, Toledo Neto J, Belucci Junior JA, et al. Avaliação da qualidade de vida dos pacientes durante o tratamento e Hanseníase. Rev. Odontologia (ATO) [Internet]. 2014 [acesso em 18 set 2018]; 14(4). Disponível em: https://www.researchgate.net/ publication/281108669 avaliacao da qualidade de vida dos pacientes durante o tratamento de hanseniase.

14. Quaggio CM da P, Virmond M, Guimarães HCQCP. Qualidade de vida da pessoa tratada da hanseníase. Hansen. Int. [Internet]. 2014 [acesso em 20 out 2018]; 39(2). Disponível em: http://www.ilsl.br/ revista/detalhe artigo.php?id=12347.

15. Conti JO, Almeida SND de, Almeida JÁ de. Prevenção de incapacidades em hanseníase: relato de caso. Salusvita [Internet]. 2013 [acesso em 17 set 2018 ]; 32(2). Disponível em: https://secure.usc.br/static/ biblioteca/salusvita/salusvita v32 n2 2013 art 04.pdf.

16. Monteiro LD, Alencar CH, Barbosa JC, Novaes CCBS, Silva RCP da, Heukelbach J. Pós-alta de hanseníase: limitação de atividade e participação social em área hiperendêmica do Norte do Brasil. Rev. bras. epidemiol. [Internet]. 2014 [acesso em 15 jun 2018]; 17(1). Disponível em: http://dx.doi. org/10.1590/1415-790X201400010008ENG.

17. D'Azevedo SSP, Freitas EM de, Nascimento LO do, Santos DCM dos, Nascimento RD do.

Percepção de pacientes com hanseníase acerca de grupos de autocuidado. Rev. enferm. UFPE on line [Internet]. 2018 [acesso em 21 jan 2019]; 12(6). Disponível em: https://periodicos.ufpe.br/revistas/ revistaenfermagem/article/viewFile/230855/29199.

18. Fleck MPA, Louzada S, Xavier M, Chachamovich E, Vieira G, Santos L, et al. Aplicação da versão em português do instrumento abreviado de avaliação da qualidade de vida "WHOQOL-bref". Rev. Saúde Públ. [Internet]. 2000 [acesso em 25 jun 2018]; 34(2). Disponível em: http://dx.doi.org/10.1590/S003489102000000200012.

19. Nobre ML, Illarramendi X, Dupnik KM, Hacker M de A, Nery JA da C, Jerônimo SMB, et al. Multibacillary leprosy by population groupsin Brazil: lessons from an observational study. PLoS Negl Trop Dis. [Internet]. 2017 [acesso em 18 set 2018]; 11(2). Disponível em: https://doi.org/10.1371/journal. pntd.0005364.

20. Conte ECM, Magalhães LCB de, Cury MR de CO, Soubhia MRC, Nardi SMT, Paschoal VDA, et al. Situação Epidemiológica da hanseníase no município de São José do Rio Preto, SP, Brasil. Arq ciênc saúde [Internet]. 2009 [acesso em 25 out 2018]; 16(4). Disponível em: http://repositorio-racs.famerp.br/ racs ol/vol-16-4/IDK1 out-dez 2010.pdf.

21. Leano HA de M, Araújo MF da A, Rodrigues RN, Bueno I de C, Lana FCF. Indicadores relacionados a incapacidade física e diagnóstico de hanseníase. Rev RENE [Internet]. 2017 [acesso em 15 ago 2018]; 18(6). Disponível em: https://doi.org/10.15253/2175-6783.2017000600018.

22. Rodrigues NC, Castro LE, Silva JG, Fontana AP, Couto Neto B, Sá VW, et al. Physical disability and it's social and functional repercussions in patients with leprosy after discharge from multidrug therapy. Lep Rev [Internet]. 2017 [acesso em 21 jan 2018]; 88. Disponível em: https://pdfs.semanticscholar.org/6a07/ b15ba4597fe63582450244770c1040b1f50c.pdf.

23. Ribeiro G de C, Lana FC. Incapacidades físicas em hanseníase: caracterização, fatores relacionados e evolução. Cogitare enferm [Internet]. 2015 [acesso em 28 jun 2018]; 20(3). Disponível em: http://dx.doi. org/10.5380/ce.v20i3.41246.

24. Santos VS, Oliveira LS, Castro FDN, Gois-Santos VT, Lemos LMD, Ribeiro M do CO, et al. Functional Activity Limitation and Quality of Life of Leprosy Cases in an Endemic Area in Northeastern Brazil. PLoS Negl. Trop. Dis. [Internet]. 2015 [acesso em 28 mar 2018]; 9(7). Disponível em: https://doi.org/10.1371/ journal.pntd.0003900. 
Recebido: 09/01/2019

Finalizado: 16/10/2019

\section{Autor Correspondente:}

Stephanie Steremberg Pires D'Azevedo

Universidade de Pernambuco

R. Arnóbio Marquês, 310 - 50.100-130 - Recife, PE, Brasil

E-mail: stephanie-steremberg@hotmail.com

\section{Contribuição dos autores:}

Contribuições substanciais para a concepção ou desenho do estudo; ou a aquisição, análise ou interpretação de dados do estudo - SSPA, DCMS, MGTA, NMN, GAA, MCVL

Elaboração e revisão crítica do conteúdo intelectual do estudo - SSPA, DCMS, GAA

Aprovação da versão final do estudo a ser publicado - SSPA, DCMS, GAA

Responsável por todos os aspectos do estudo, assegurando as questões de precisão ou integridade de qualquer parte do estudo - SSPA, DCMS 